\title{
TRANSITIVITY OF EUCLIDEAN-TYPE EXTENSIONS OF HYPERBOLIC SYSTEMS
}

\author{
I. MELBOURNE, V. NIŢICĂ, A. TÖRÖK
}

\begin{abstract}
Let $f: X \rightarrow X$ be the restriction to a hyperbolic basic set of a smooth diffeomorphism. We show that in the class of $C^{r}, r>0$, cocycles with fiber special Euclidean group $S E(n)$ those that are transitive form a residual set (countable intersection of open dense sets). This result is new for $n \geq 3$ odd.

More generally, we consider Euclidean-type groups $G \ltimes \mathbb{R}^{n}$ where $G$ is a compact connected Lie group acting linearly on $\mathbb{R}^{n}$. When Fix $G=\{0\}$, it is again the case that the transitive cocycles are residual. When Fix $G \neq\{0\}$, the same result holds on restriction to the subset of cocycles that avoid an obvious and explicit obstruction to transitivity.
\end{abstract}

\section{INTRODUCTION}

In this paper we continue to study topological transitivity in various classes of noncompact group-extensions of hyperbolic systems. Consider a continuous transformation $f: X \rightarrow X$, a Lie group $\Gamma$, and a continuous map $\beta: X \rightarrow \Gamma$ called a cocycle. These determine a skew product, or $\Gamma$-extension,

$$
f_{\beta}: X \times \Gamma \rightarrow X \times \Gamma, \quad f_{\beta}(x, \gamma)=(f x, \gamma \beta(x)) .
$$

It is assumed throughout the paper that $X$ is a hyperbolic basic set. The $\Gamma$-extension $f_{\beta}$ is called topologically transitive, or simply transitive, if it has a dense orbit. Of interest to us is whether noncompact Lie group extensions of a hyperbolic basic set are typically topologically transitive.

Let $\left(M, d_{M}\right)$ be a smooth manifold endowed with a Riemannian metric. Let $f$ : $M \rightarrow M$ be a smooth diffeomorphism and $X \subset M$ a compact and $f$-invariant subset of $M$. We say that $X$ is hyperbolic if there exists a continuous $D f$-invariant splitting $E^{s} \oplus E^{u}$ of the tangent bundle $T_{X} M$ and constants $C_{1}>0,0<\lambda<1$, such that for all $n \geq 0$ and $x \in X$ we have:

$$
\begin{aligned}
\left\|\left(D f^{n}\right)_{x} v\right\| & \leq C_{1} \lambda^{n}\|v\|, \quad v \in E_{x}^{s} \\
\left\|\left(D f^{-n}\right)_{x} v\right\| & \leq C_{1} \lambda^{n}\|v\|, \quad v \in E_{x}^{u} .
\end{aligned}
$$

Date: August 3, 2008.

1991 Mathematics Subject Classification. 37D20, 37D30.

Key words and phrases. Noncompact group extension, stable transitivity, skew-product, hyperbolic basic set.

This research was supported in part by a Leverhulme Fellowship (IM), by NSF Grant DMS0500832 (VN), by NSF Grant DMS-0244529 and DMS-0600927 (AT) and by EPSRC Grant GR/S11862/01 (IM,VN,AT). 
We say that $X$ is locally maximal if there exists an open neighborhood $U$ of $X$ such that every compact $f$-invariant set of $U$ is contained in $X$. A locally maximal hyperbolic set $X$ is a basic set for $f: M \rightarrow M$ if $f: X \rightarrow X$ is transitive and $X$ does not consist of a single periodic orbit.

Given a connected Lie group $\Gamma$ and a cocycle $\beta: X \rightarrow \Gamma$, we consider the $\Gamma$ extension $f_{\beta}: X \times \Gamma \rightarrow X \times \Gamma$ given by $f_{\beta}(x, \gamma)=(f x, \gamma \beta(x))$. For brevity, we say that the cocycle $\beta$ is transitive if the $\Gamma$-extension $f_{\beta}$ is transitive. In [5] we proposed a general conjecture about transitivity in the class of Hölder cocycles: namely that modulo obstructions appearing from the fact that the range of the cocycle is included in a semigroup, transitivity is open and dense. The conjecture is proved for various classes of Lie groups, mostly semidirect products of compact and Euclidean, in [2, 4, [5, 6. 8]. In addition [5] exhibits open sets of $C^{r}$ transitive cocycles with fiber $S p(n)$.

An important test case is presented by cocycles with fiber the special Euclidean group $S E(n)=S O(n) \ltimes \mathbb{R}^{n}$. It is shown in [4, 5, 6] that when $n$ is even the set of cocycles that are transitive is Hölder-open and $C^{r}$-dense. The conjecture remains unsolved for $n \geq 3$ odd.

Here we show that for $S E(n), n \geq 3$ odd, the transitive $C^{r}$ cocycles form a residual subset (actually, by Remark 1.3(b), a countable intersection of open dense subsets) of the space of all $C^{r}$ cocycles for all $r>0$. In other words, transitivity is $C^{r}$-generic for such extensions. The proof introduces a number of new ideas.

Theorem 1.1. Let $X$ be a basic hyperbolic set for $f: X \rightarrow X$. Let $r>0$ and let $n \geq 3$ be odd. Amongst the $C^{r}$ cocycles $\beta: X \rightarrow S E(n)$, the transitive cocycles form a residual set.

More generally, we consider Euclidean-type groups of the form $\Gamma=G \ltimes \mathbb{R}^{n}$ where $G$ is a compact connected Lie group acting linearly (and orthogonally) on $\mathbb{R}^{n}$ and the group multiplication is given by

$$
\left(g_{1}, v_{1}\right)\left(g_{2}, v_{2}\right)=\left(g_{1} g_{2}, v_{1}+g_{1} v_{2}\right)
$$

Let Fix $G=\left\{v \in \mathbb{R}^{n}: g v=v\right.$ for all $\left.g \in G\right\}$. Set $\pi: \Gamma \rightarrow$ Fix $G$ to be the projection onto the $\mathbb{R}^{n}$-component and then orthogonal projection onto Fix $G$. If Fix $G \neq\{0\}$, then there is an obvious obstruction to transitivity namely that $\pi \beta: X \rightarrow$ Fix $G$ takes values in a halfspace. Recall that two cocycles $\beta, \beta^{\prime}: X \rightarrow \mathbb{R}^{d}$ are said to be cohomologous if there exists $P: X \rightarrow \mathbb{R}^{d}$ continuous such that $\beta^{\prime}(x)=P(f x)+$ $\beta(x)-P(x), x \in X$. More generally, if $\pi \beta$ is cohomologous to a cocycle with values in a half space, then $f_{\beta}$ is not transitive. This is the only obstruction in generalising Theorem 1.1 to general Euclidean-type groups.

Theorem 1.2. Let $X$ be a basic hyperbolic set for $f: X \rightarrow X$ and let $\Gamma=G \ltimes \mathbb{R}^{n}$ be a Euclidean-type group. Let $r>0$. Define $\mathcal{S}$ to be the space of $C^{r}$ cocycles $\beta: X \rightarrow \Gamma$ for which $\pi \beta: X \rightarrow$ Fix $G$ is not cohomologous to a cocycle with values in a halfspace.

Then $\mathcal{S}$ is an open subset of the space of $C^{r}$ cocycles, and the transitive cocycles $\beta: X \rightarrow \Gamma$ form a residual subset of $\mathcal{S}$.

Remark 1.3. (a) If Fix $G=0$, then there is no obstruction to transitivity, so Theorem 1.1 is a special case of Theorem 1.2 . 
(b) By a standard argument, the set of transitive $C^{r}$ cocycles can be written as a countable intersection of $C^{r}$-open sets. Hence it suffices to prove density in Theorems 1.1 and 1.2. We include the argument below:

Choose a countable basis $\left\{U_{k}\right\}_{k}$ of the topology on $X \times \Gamma$ and denote by $C_{k, \ell}^{r}$ the $C^{r}$ cocycles $\beta \in \mathcal{S}$ for which there is an integer $n$ such that $f_{\beta}^{n}\left(U_{k}\right) \cap U_{\ell} \neq \emptyset$. Each set $C_{k, \ell}^{r}$ is clearly $C^{r}$-open, and $f$ is transitive if and only if $\beta$ is in each of the sets $C_{k, \ell}^{r}$.

Let $G \ltimes \mathbb{R}^{n}$ be a Euclidean-type group. We can write $\mathbb{R}^{n}$ as a sum of $G$-irreducible representations $V_{\ell}$ which we divide into three types:

Class I: $V_{\ell} \not \subset$ Fix $G$ and $V_{\ell} \cap$ Fix $g \neq\{0\}$ for all $g \in G$.

Class II: $V_{\ell} \subset \operatorname{Fix} G$.

Class III: $V_{\ell} \cap \operatorname{Fix} g=\{0\}$ for some $g \in G$.

(Here, Fix $g=\left\{v \in \mathbb{R}^{n}: g v=v\right\}$.)

We say that the Euclidean-type group $G \ltimes \mathbb{R}^{n}$ is of class $I$ if all the $G$-irreducible representations $V_{\ell}$ are of class I. This includes the groups in Theorem 1.1. The main task in this paper is to prove Theorem 1.2 for the class I groups. The general result is then proved by incorporating ideas of [4, 8].

The remainder of the paper is organized as follows. In Section 2, we recall some general results from [5]. In Section 3, we introduce a new construction that seems particularly useful for cocycles that satisfy a subexponential growth condition. In Section 4, this construction is specialized to the setting of Euclidean-type groups. A second key new idea for Euclidean-type extensions appears in Section 5. The proof of Theorem 1.2 is given for class I groups in Section 6 and for general Euclidean-type groups in Section 7 .

\section{CRITERION FOR TRANSITIVITY}

Let $\Gamma$ be a connected Lie group with Lie algebra $L \Gamma$. We denote by $e_{\Gamma}$ the identity element of $\Gamma$. Let Ad denote the adjoint representation of $\Gamma$ on $L \Gamma$. Let $\|\cdot\|$ be a norm on $L \Gamma$. There is a metric $d$ on $\Gamma$ with the following properties (see Pollicott and Walkden [10]):

(1) $d\left(\gamma \gamma_{1}, \gamma \gamma_{2}\right)=d\left(\gamma_{1}, \gamma_{2}\right)$;

(2) $d\left(\gamma_{1} \gamma, \gamma_{2} \gamma\right) \leq\|\operatorname{Ad}(\gamma)\| d\left(\gamma_{1}, \gamma_{2}\right)$;

for any $\gamma, \gamma_{1}, \gamma_{2} \in \Gamma$.

Definition 2.1. Let $f: X \rightarrow X$ be a map and $\beta: X \rightarrow \Gamma$ a cocycle. For $k \geq 1$, we write $f_{\beta}^{k}(x, \gamma)=\left(f^{k} x, \gamma \beta(k, x)\right)$ where

$$
\beta(k, x)=\beta(x) \beta(f x) \cdots \beta\left(f^{k-1} x\right)=\prod_{j=0}^{k-1} \beta\left(f^{j} x\right) .
$$

(Occasionally, we use the last formula to keep notation simple; its meaning is the ordered product given by the middle expression).

If $Q$ is a trajectory of $f$ of length $k$ (i.e. $Q=\left\{x, f(x), \ldots, f^{k-1}(x)\right\}$ for some $x$ ), then we define the height of $\beta$ over $Q$ to be $\beta(Q)=\beta(k, x)$. In particular, if $x$ is a 
periodic point of period $\ell$, then the height of the corresponding periodic orbit $P$ is $\beta(P)=\beta(\ell, x)$.

By abuse of notation, we often refer to "the periodic orbit $P$ " instead of "the orbit of the periodic point $x$ " when $x$ is clear from the context.

Definition 2.2. Given a cocycle $\beta: X \rightarrow \Gamma$ over $f: X \rightarrow X$, define $\mu \geq 1$ to be

$$
\mu=\max \left\{\lim _{n \rightarrow \infty} \sup _{x \in X}\|\operatorname{Ad}(\beta(n, x))\|^{1 / n}, \lim _{n \rightarrow \infty} \sup _{x \in X}\left\|\operatorname{Ad}(\beta(n, x))^{-1}\right\|^{1 / n}\right\} .
$$

For $f$ fixed, we say that the cocycle $\beta$ has subexponential growth if $\mu=1$.

Remark 2.3. The subexponential growth condition is automatically satisfied for any cocycle if the group $\Gamma$ is compact, nilpotent, or a semidirect product of compact and nilpotent. In particular, cocycles with values in Euclidean-type groups have subexponential growth.

One of the key notions used in this paper was introduced in [5]:

Definition 2.4. Let $\Gamma$ be a connected Lie group, $X$ a basic hyperbolic set for $f$ : $X \rightarrow X, \beta: X \rightarrow \Gamma$ a cocycle, and $f_{\beta}: X \times \Gamma \rightarrow X \times \Gamma$ the skew-extension. Given $x \in X$, let

$$
\begin{array}{r}
\mathcal{L}_{\beta}(x)=\left\{\gamma \in \Gamma: \text { there exist } x_{k} \in X \text { and } n_{k}>0\right. \text { such that } \\
\left.\qquad x_{k} \rightarrow x \text { and } f_{\beta}^{n_{k}}\left(x_{k}, e_{\Gamma}\right) \rightarrow(x, \gamma)\right\} .
\end{array}
$$

That is, the set $\mathcal{L}_{\beta}(x)$ consists of the possible limits $\lim _{k \rightarrow \infty} \beta\left(n_{k}, x_{k}\right)$, subject to $x_{k} \rightarrow x$ and $f^{n_{k}}\left(x_{k}\right) \rightarrow x$. Note that we do not require that $n_{k} \rightarrow \infty$ or that $x_{k} \neq x$. Clearly $\mathcal{L}_{\beta}(x)$ is a closed subset of $\Gamma$.

The following theorem is a special case of [5, Lemma 3.1, Theorem 3.3].

Theorem 2.5. Assume that $X$ is a hyperbolic basic set for $f: X \rightarrow X$, that $\Gamma$ is a connected Lie group and $\beta: X \rightarrow \Gamma$ a Hölder cocycle that has subexponential growth. Then

(a) $\mathcal{L}_{\beta}(x)$ is a closed semigroup of $\Gamma$ for each $x \in X$.

(b) If there exists a point $x_{0} \in X$ such that $\mathcal{L}_{\beta}\left(x_{0}\right)=\Gamma$ then $\beta$ is a transitive cocycle.

Recall that $W^{s}(x)$ and $W^{u}(x)$ denote the stable and unstable leaves of $f$ through $x$. The next lemma is a consequence of [9, Appendix A].

Lemma 2.6. Assume that $X$ is a hyperbolic basic set for $f: X \rightarrow X$, that $\Gamma$ is a connected Lie group and $\beta: X \rightarrow \Gamma$ an $\alpha$-Hölder cocycle that has subexponential growth. Then the $\Gamma$-extension $f_{\beta}: X \times \Gamma \rightarrow X \times \Gamma$ admits stable and unstable foliations which are $\alpha$-Hölder and invariant under right multiplication by elements of $\Gamma$. The stable and unstable leaves of $f_{\beta}$ through $\left(x, e_{\Gamma}\right) \in X \times \Gamma$ are the graphs of the functions

$$
\begin{aligned}
& \gamma_{x}^{s}: W^{s}(x) \rightarrow \Gamma, \quad \gamma_{x}^{s}(y)=\lim _{n \rightarrow \infty} \beta(n, x) \beta(n, y)^{-1} \\
& \gamma_{x}^{u}: W^{u}(x) \rightarrow \Gamma, \quad \gamma_{x}^{u}(y)=\lim _{n \rightarrow \infty} \beta(-n, x) \beta(-n, y)^{-1}
\end{aligned}
$$


These functions are $\alpha$-Hölder and vary continuously with the cocycle $\beta$ in the following sense: if $\beta_{k} \rightarrow \beta$ in $C^{0}$-topology and $\beta_{k}$ remains $C^{\alpha}$-bounded, then on $W_{l o c}^{s}(x), \gamma_{k, x}^{s} \rightarrow$ $\gamma_{x}^{s}$ and $\gamma_{k, x}^{u} \rightarrow \gamma_{x}^{u}$ in $C^{0}$-topology.

We call the values of the functions $\gamma_{x}^{s}, \gamma_{x}^{u}$ holonomies along stable/unstable leaves. The following lemma is a special case of [5, Lemma 2.2].

Lemma 2.7. Assume that $X$ is a hyperbolic basic set for $f: X \rightarrow X$, that $\Gamma$ is a connected Lie group and $\beta: X \rightarrow \Gamma$ a $\alpha$-Hölder cocycle that has subexponential growth. Then there is a constant $C_{5}>0$ with the following property.

Given $\varepsilon>0$ sufficiently small and $n \geq 1$, assume that there are two trajectories $x_{k}=f^{k}\left(x_{0}\right), y_{k}=f^{k}\left(y_{0}\right)$, such that $d_{M}\left(x_{k}, y_{k}\right)<\varepsilon$ for $0 \leq k \leq n-1$. Then

$$
d\left(\beta\left(n, x_{0}\right), \beta\left(n, y_{0}\right)\right) \leq C_{5}\left(\left\|\operatorname{Ad}\left(\beta\left(n, x_{0}\right)\right)\right\|+1\right) \varepsilon^{\alpha} .
$$

Moreover, if $\Gamma$ is compact, then

$$
d\left(\beta\left(n, x_{0}\right), \beta\left(n, y_{0}\right)\right) \leq C_{5} \varepsilon^{\alpha} .
$$

\section{Admissible sequences of PRoducts of holonomies}

Throughout this section, $\left(M, d_{M}\right)$ is Riemannian manifold, $X \subset M$ is a basic hyperbolic set for $f: X \rightarrow X$ with contraction constant $\lambda \in(0,1)$ satisfying (1.1), $\Gamma$ a connected Lie group and $\beta: X \rightarrow \Gamma$ a $\alpha$-Hölder cocycle that has subexponential growth.

Definition 3.1. By a periodic heteroclinic cycle we mean a cycle consisting of points $p_{1}, \ldots, p_{k}$ that are periodic for the map $f$, have disjoint trajectories, and such that $p_{j}$ is transverse heteroclinic to $p_{j+1}$ through a point $\zeta_{j} \in W^{u}\left(p_{j}\right) \cap W^{s}\left(p_{j+1}\right)$, for $j=1, \ldots, k$ (where $\left.p_{k+1}=p_{1}\right)$.

Let $P_{1}, \ldots, P_{k}$ be the corresponding periodic orbits and denote the periods by $\ell_{1}, \ldots, \ell_{k}$. Denote by $O_{j}$ the heteroclinic trajectory from $p_{j}$ to $p_{j+1}$ (of the point $\zeta_{j}$ chosen above), and by $H_{j}$ the holonomy along this heteroclinic connection (that is, along $W^{u}\left(p_{j}\right)$ from $p_{j}$ to $\zeta_{j}$ and then along $W^{s}\left(p_{j+1}\right)$ from $\zeta_{j}$ to $\left.p_{j+1}\right)$.

Replace the heteroclinic orbit $O_{j}$ from $p_{j}$ to $p_{j+1}$ by the trajectory $Q_{j}$ of length $\ell_{j} M_{j}+\ell_{j+1} M_{j+1}$ that spends time $\ell_{j} M_{j}$ in the first half of $O_{j}$ and time $\ell_{j+1} M_{j+1}$ in the second half of $O_{j}$; that is, $Q_{j}=\left\{f^{n}\left(\zeta_{j}\right) \mid-\ell_{j} M_{j} \leq n<0\right\} \cup\left\{f^{n}\left(\zeta_{j}\right) \mid 0 \leq n<\right.$ $\left.\left.\ell_{j+1} M_{j+1}\right\}\right)$. For the trajectory connecting $p_{k}$ to $p_{k+1}$, we allow $M_{1}$ and $M_{k+1}$ to be distinct. The positive integers $M_{j}$ will be chosen later.

Consider the heights $\beta\left(P_{j}\right)$ and $\beta\left(Q_{j}\right)$ over the periodic orbits $P_{j}$ and trajectories $Q_{j}$ (see Definition 2.1).

Lemma 3.2. For $j=1, \ldots, k$, the limit

$$
\lim _{M_{j}, M_{j+1} \rightarrow \infty} \beta\left(P_{j}\right)^{-M_{j}} \beta\left(Q_{j}\right) \beta\left(P_{j+1}\right)^{-M_{j+1}}=H_{j}
$$

exists and is the product of the holonomies along the unstable and stable leaves of $O_{j}$, from $p_{j}$ to $p_{j+1}$. 
Proof. This follows from Lemma 2.6.

Definition 3.3. Consider a sequence of vectors $N(1), N(2) \ldots \in \mathbb{N}^{k+1}$ whose entries are positive integers. Write $N(i)=\left(M_{1}(i), \ldots, M_{k+1}(i)\right)$. The sequence is admissible if there is a constant $C_{2} \geq 1$ such that $M_{p}(i) / M_{q}(i) \leq C_{2}$ for all $p, q=1 \ldots, k+1$ and all $i \geq 1$.

If $N=\left(M_{1}, \ldots, M_{k+1}\right)$ is a sequence of vectors, we write $N \rightarrow \infty$ if $M_{p} \rightarrow \infty$ for each $p=1, \ldots, k+1$. (For an admissible sequence, it is equivalent that $M_{p} \rightarrow \infty$ for at least one value of $p$.)

Theorem 3.4. Let $N=\left(M_{1}, \ldots, M_{k+1}\right) \in \mathbb{N}^{k+1}$. Define

$$
A(N)=\beta\left(P_{1}\right)^{M_{1}} H_{1} \beta\left(P_{2}\right)^{2 M_{2}} H_{2} \cdots \beta\left(P_{k}\right)^{2 M_{k}} H_{k} \beta\left(P_{1}\right)^{M_{k+1}} .
$$

If the limit $A=\lim _{N \rightarrow \infty} A(N)$ exists along an admissible sequence $N(1), N(2), \ldots$, then $A \in \mathcal{L}_{\beta}\left(p_{1}\right)$.

Remark 3.5. We can rewrite the expression $A(N)$ as

$$
A(N)=\bar{\beta}_{1}^{M_{1}} \bar{\beta}_{2}^{2 M_{2}} \cdots \bar{\beta}_{k}^{2 M_{k}} \bar{\beta}_{k+1}^{M_{k+1}} \bar{H}_{k+1},
$$

where $\bar{H}_{1}=e_{\Gamma}, \bar{H}_{j}=H_{1} H_{2} \cdots H_{j-1}$ for $j=2, \ldots, k+1$, and $\bar{\beta}_{j}=\bar{H}_{j} \beta\left(P_{j}\right) \bar{H}_{j}^{-1}$. (Note that $\bar{\beta}_{k+1}$ is related to $\bar{\beta}_{1}$ but the remaining $\bar{\beta}_{j}$ s can be modified independently.)

In the remainder of this section, we prove Theorem 3.4. From now on we assume for notational simplicity that $P_{j}=p_{j}$ are fixed points $\left(\right.$ so $\ell_{j}=1$ ).

Given $N=\left(M_{1}, \ldots, M_{k+1}\right) \in \mathbb{N}^{k+1}$, define

$$
\begin{aligned}
|N| & =\left(M_{1}+M_{k+1}\right) / 2+\sum_{j=2}^{k} M_{j}, \\
\min N & =\min \left\{M_{1}, \ldots, M_{k+1}\right\}, \quad \max N=\max \left\{M_{1}, \ldots, M_{k+1}\right\} .
\end{aligned}
$$

Note that for an admissible sequence $N$, we have $\max N \leq C_{2} \min N$.

Define

$$
H_{j}(N)=\beta\left(P_{j}\right)^{-M_{j}} \beta\left(Q_{j}\right) \beta\left(P_{j+1}\right)^{-M_{j+1}} .
$$

By Lemma 3.2. $\lim _{N \rightarrow \infty} H_{j}(N)=H_{j}$ (independent of the sequence $N$ ). Moreover, by [9, proof of Theorem $4.3(\mathrm{~g})]$, there is $\delta_{0} \in(0,1)$ such that

$$
d\left(H_{j}(N), H_{j}\right)=O\left(\delta_{0}^{\min N}\right) .
$$

Recall that $Q_{j}$ is a trajectory of length $M_{j}+M_{j+1}$ that shadows the heteroclinic connection from $p_{j}$ to $p_{j+1}$. Concatenate these trajectories to form a periodic pseudoorbit $Q=Q_{1} \ldots Q_{k}$ of length $2|N|$. Then $Q$ is a $\delta$-pseudo-orbit with $\delta \leq C_{3} \lambda^{\min N}$, where $C_{3}>0$ is a constant (depending on $f: X \rightarrow X$ ) and $\lambda$ is the contraction constant. By hyperbolicity of $X$, there is a periodic orbit $\tilde{Q}$ of length $2|N|$ that $\varepsilon$-shadows $Q$ with $\varepsilon \leq C_{4} \lambda^{\min N}$, where $C_{4}>0$ is a constant. See [7, page 74] for standard shadowing techniques.

Proposition 3.6. 
(a) $\beta(Q)=\beta\left(P_{1}\right)^{M_{1}} H_{1}(N) \beta\left(P_{2}\right)^{2 M_{2}} H_{2}(N) \cdots \beta\left(P_{k}\right)^{2 M_{k}} H_{k}(N) \beta\left(P_{1}\right)^{M_{k+1}}$.

(b) $\lim _{N \rightarrow \infty} d(\beta(Q), \beta(\tilde{Q}))=0$ along admissible sequences $N$.

(c) $\lim _{N \rightarrow \infty} d(\beta(Q), A(N))=0$ along admissible sequences $N$.

Proof. Part (a) is a direct calculation, namely

$$
\beta(Q)=\prod_{j=1}^{k} \beta\left(Q_{j}\right)=\prod_{j=1}^{k} \beta\left(P_{j}\right)^{M_{j}} H_{j}(N) \beta\left(P_{j+1}\right)^{M_{j+1}} .
$$

Next, write $\tilde{Q}=\tilde{Q}_{1} \ldots \tilde{Q}_{k}$ where $\tilde{Q}_{j}$ has length $M_{j}+M_{j+1}$. Define $\gamma_{j}=\beta\left(Q_{j}\right), \tilde{\gamma}_{j}=$ $\beta\left(\tilde{Q}_{j}\right)$. Note that $Q_{j}$ and $\tilde{Q}_{j}$ have length at most $2 \max N$, and that $\tilde{Q}_{j} \varepsilon$-shadows $Q_{j}$ with $\varepsilon \leq C_{4} \lambda^{\min N}$. It follows from Lemma 2.7 that $d\left(\gamma_{i}, \tilde{\gamma}_{i}\right) \leq C \lambda^{\alpha \min N}\left(\left\|\operatorname{Ad}\left(\gamma_{i}\right)\right\|+1\right)$ where $C=C_{4}^{\alpha} C_{5}$. Hence, using the properties of the metric on $\Gamma$ and the fact that $\beta$ has subexponential growth, we have

$$
\begin{aligned}
d(\beta(Q), \beta(\tilde{Q}))= & d\left(\gamma_{1} \gamma_{2} \cdots \gamma_{k}, \tilde{\gamma}_{1} \tilde{\gamma}_{2} \cdots \tilde{\gamma}_{k}\right) \\
\leq & d\left(\gamma_{1} \gamma_{2} \cdots \gamma_{k}, \tilde{\gamma}_{1} \gamma_{2} \cdots \gamma_{k}\right)+d\left(\tilde{\gamma}_{1} \gamma_{2} \gamma_{3} \cdots \gamma_{k}, \tilde{\gamma}_{1} \tilde{\gamma}_{2} \gamma_{3} \cdots \gamma_{k}\right)+ \\
& \cdots+d\left(\tilde{\gamma}_{1} \tilde{\gamma}_{2} \cdots \tilde{\gamma}_{k-1} \gamma_{k}, \tilde{\gamma}_{1} \tilde{\gamma}_{2} \cdots \tilde{\gamma}_{k-1} \tilde{\gamma}_{k}\right) \\
\leq & d\left(\gamma_{1}, \tilde{\gamma}_{1}\right)\left\|\operatorname{Ad}\left(\gamma_{2} \cdots \gamma_{k}\right)\right\|+d\left(\gamma_{2}, \tilde{\gamma}_{2}\right)\left\|\operatorname{Ad}\left(\gamma_{3} \cdots \gamma_{k}\right)\right\|+\cdots+d\left(\gamma_{k}, \tilde{\gamma}_{k}\right) \\
\leq & C \lambda^{\alpha \min N}\left[(1+\eta)^{2 \max N}+1\right]\left[(1+\eta)^{2 \max N}+\cdots+(1+\eta)^{2(k-1) \max N}\right],
\end{aligned}
$$

where $\eta>0$ can be chosen arbitrarily small and $\|\operatorname{Ad}(\beta(n, x))\| \leq(1+\eta)^{n}$ for $n$ large enough. Restricting to admissible sequences, $\min N$ and $\max N$ are comparable and part (b) follows. The proof of part (c) is similar using (3.1).

Proof of Theorem 3.4. By assumption, $A(N) \rightarrow A$. Hence by Proposition 3.6(b,c), $\beta(\tilde{Q}) \rightarrow A$. We conclude that $A \in \mathcal{L}_{\beta}\left(p_{1}\right)$ by definition of $\mathcal{L}_{\beta}\left(p_{1}\right)$.

\section{Construction for Euclidean-type Groups}

In this section, we specialise the construction in Section 3 to the case of the Euclidean-type group $\Gamma=G \ltimes \mathbb{R}^{n}$. We denote the identity element in $G$ by $e_{G}$. Define $\bar{\beta}_{j}$ as in Remark 3.5 and write

$$
\bar{\beta}_{j}=\left(g_{j}, v_{j}\right), \quad j=1, \ldots, k+1 .
$$

For each $j$, we have the orthogonal decomposition $\mathbb{R}^{n}=\operatorname{Fix} g_{j} \oplus\left(\operatorname{Fix} g_{j}\right)^{\perp}$ where $\operatorname{Fix} g_{j}=\operatorname{ker}\left(g_{j}-I\right)$. Write $v_{j}=w_{j} \oplus w_{j}^{\prime}$ where $w_{j} \in \operatorname{Fix} g_{j}$ and $w_{j}^{\prime} \in\left(\operatorname{Fix} g_{j}\right)^{\perp}$.

Define

and let

$$
h_{j}=g_{1}^{n_{1}} g_{2}^{2 n_{2}} \ldots g_{j-1}^{2 n_{j-1}}, \quad u_{j}=h_{j} w_{j}
$$

$$
Z\left(u_{1}, \ldots, u_{k+1}\right)=\left\{\alpha_{1} u_{1}+\cdots+\alpha_{k+1} u_{k+1}: \alpha_{1}, \ldots, \alpha_{k+1}>0\right\} \subset \mathbb{R}^{n} .
$$

Theorem 4.1. Assume that

(1) $g_{j}$ has finite order for each $j=1, \ldots, k$; and

(2) $n_{1}, \ldots, n_{k}$ are integers such that $0 \in Z\left(u_{1}, \ldots, u_{k+1}\right)$. 
Then

$$
\begin{aligned}
A & =\beta\left(P_{1}\right)^{n_{1}} H_{1} \beta\left(P_{2}\right)^{2 n_{2}} H_{2} \cdots \beta\left(P_{k}\right)^{2 n_{k}} H_{k} \beta\left(P_{1}\right)^{n_{k+1}} \\
& =\bar{\beta}_{1}^{n_{1}} \bar{\beta}_{2}^{2 n_{2}} \cdots \bar{\beta}_{k}^{2 n_{k}} \bar{H}_{k+1} \in \mathcal{L}_{\beta}\left(p_{1}\right) .
\end{aligned}
$$

Proof. Choose $M_{j}$ such that $g_{j}^{M_{j}}=e_{G}$ and define $N=\left(M_{1}+n_{1}, \ldots, M_{k}+n_{k}, M_{k+1}\right)$. A calculation shows that

$$
\begin{aligned}
A(N) & =\left(g_{1}, v_{1}\right)^{M_{1}+n_{1}}\left(g_{2}, v_{2}\right)^{2 M_{2}+2 n_{2}} \ldots\left(g_{k}, v_{k}\right)^{2 M_{k}+2 n_{k}}\left(g_{k+1}, v_{k+1}\right)^{M_{k+1}} \bar{H}_{k+1} \\
& =\left(e_{G}, w(N)\right)\left(g_{1}, v_{1}\right)^{n_{1}}\left(g_{2}, v_{2}\right)^{2 n_{2}} \ldots\left(g_{k}, v_{k}\right)^{2 n_{k}} \bar{H}_{k+1}=\left(e_{G}, w(N)\right) A,
\end{aligned}
$$

where $w(N)=M_{1} u_{1}+2 M_{2} u_{2}+\cdots+2 M_{k} u_{k}+M_{k+1} u_{k+1}$.

We claim that there is an admissible sequence $N$ satisfying the above constraints such that $\lim _{N \rightarrow \infty} w(N)=0$. Then $A(N) \rightarrow A$, and so it follows from Theorem 3.4 that $A \in \mathcal{L}_{\beta}\left(p_{1}\right)$.

To prove the claim, we repeat an argument used in [6, Lemma 2.12]. By condition (2), there exist $\alpha_{1}, \ldots, \alpha_{k+1}>0$ such that $\alpha_{1} u_{1}+2 \sum_{j=2}^{k} \alpha_{j} u_{j}+\alpha_{k+1} u_{k+1}=0$. Hence $t \alpha_{1} u_{1}+2 \sum_{j=2}^{k} t \alpha_{j} u_{j}+t \alpha_{k+1} u_{k+1}=0$ for each $t>0$, and there is a sequence $t_{i} \rightarrow \infty$ such that the fractional part of $t_{i} \alpha_{j}$ converges to zero for each $j$. Let $M_{j}(i)=q\left[t_{i} \alpha_{j}\right]$ where $q$ is the least common multiple of the orders of the $g_{j}$ s. Then $N(i)=\left(M_{1}(i)+n_{1}, \ldots, M_{k}(i)+n_{k}, M_{k+1}(i)\right)$ is the required admissible sequence.

Corollary 4.2. Suppose that the hypotheses of Theorem 4.1 are valid and $w$ lies in the semigroup generated by $u_{1}, 2 u_{2}, \ldots, 2 u_{k}, u_{k+1}$. Then $\left(e_{G}, q w\right) A \in \mathcal{L}_{\beta}\left(p_{1}\right)$ where $q$ is the least common multiple of the orders of the $g_{j} s$.

Proof. Let $M_{j}(i)=q\left[t_{i} \alpha_{j}\right]$ be as in the proof of Theorem 4.1 and choose $m_{1} \ldots, m_{k+1}$ such that $w=m_{1} u_{1}+2 \sum_{j=2}^{k} m_{j} u_{j}+m_{k+1} u_{k+1}$. Define $N(i)=\left(M_{1}(i)+q m_{1}+\right.$ $\left.n_{1}, \ldots, M_{k}(i)+q m_{k}+n_{k}, M_{k+1}(i)+q m_{k+1}\right)$. Then $N(i)$ is an admissible sequence and $A(N) \rightarrow\left(e_{G}, q w\right) A$.

\section{Perturbing the heteroclinic Cycle}

Let $\Gamma=G \ltimes \mathbb{R}^{n}$ be a Euclidean-type group. Write $\beta: X \rightarrow \Gamma$ as $\beta=\left(\beta_{G}, \beta_{\mathbb{R}^{n}}\right)$ : $X \rightarrow G \ltimes \mathbb{R}^{n}$. By [2], the compact group extension $f_{\beta_{G}}: X \times G \rightarrow X \times G$ is transitive for an open dense set of $C^{r}$ cocycles $\beta: X \rightarrow \Gamma$.

In this section we show that we can specify the $G$-component of a particular $\bar{\beta}_{j}$ without significantly changing the remaining $\bar{\beta}_{j}$ s by modifying the heteroclinic cycle $p_{1}, \ldots, p_{k}$. (The cocycle itself is unchanged during this modification of the heteroclinic cycle.)

Lemma 5.1. Let $\Gamma=G \ltimes \mathbb{R}^{n}$ be a Euclidean-type group. Assume that the compact group extension $f_{\beta_{G}}: X \times G \rightarrow X \times G$ is transitive. Suppose that $p_{1}, \ldots, p_{k}$ is a periodic heteroclinic cycle in $X$ with associated elements $\bar{\beta}_{j}=\left(g_{j}, v_{j}\right)$ in (4.1), $j=1, \ldots, k+1$. 
Let $j_{0} \in\{2, \ldots, k\}, r>0$, and $\bar{g} \in G$. Then there is a periodic point $p_{j_{0}}^{\prime}$ arbitrarily close to $p_{j_{0}}$ such that the periodic heteroclinic cycle $p_{1}^{\prime}, p_{2}^{\prime}, \ldots, p_{k}^{\prime}$ obtained by replacing $p_{j_{0}}$ with $p_{j_{0}}^{\prime}$ has associated elements $\bar{\beta}_{j}^{\prime}=\left(g_{j}^{\prime}, v_{j}^{\prime}\right)$ that satisfy:

(i) $d\left(\bar{\beta}_{j}^{\prime}, \bar{\beta}_{j}\right)<r$ for $j=1, \ldots, k+1, j \neq j_{0}$, and

(ii) $d\left(g_{j_{0}}^{\prime}, \bar{g}\right)<r$.

Note that for the new cycle $p_{1}^{\prime}, p_{2}^{\prime}, \ldots, p_{k}^{\prime}$ we keep the same heteroclinic points, $\zeta_{j}^{\prime}=\zeta_{j}$, except for $\zeta_{j_{0}-1}^{\prime}$ and $\zeta_{j_{0}}^{\prime}$ which are obtained by continuity using the new $p_{j_{0}}^{\prime}$.

Proof. By compactness, there exists a constant $K \geq 1$ such that $\|\operatorname{Ad}(g)\| \leq K$ for all $g \in G$. Recall that $\bar{H}_{j}=H_{1} H_{2} \ldots H_{j-1} \in \Gamma$ is the product of holonomies associated with the heteroclinic trajectories between $p_{1}, p_{2}, \ldots, p_{j}$. Let $h$ be the $G$-component of $\bar{H}_{j_{0}}$ and define $\hat{g}=h^{-1} \bar{g} h$.

Let $\delta>0$. By transitivity of $X \times G$, there exists $x \in X$ and an integer $m \geq 1$ such that $x$ and $f^{m} x$ are $\delta$-close to $p_{j_{0}}$ and $d\left(\beta_{G}(m, x), \hat{g}\right)<r / 4$. By Anosov's closing lemma [3], there is a periodic point $p_{j_{0}}^{\prime}$ of period $m$ such that $d_{M}\left(f^{i} p_{j_{0}}^{\prime}, f^{i} x\right)<C \delta$ for $i=0, \ldots, m$, where $C$ is independent of the periodic orbit. We can arrange that $p_{j_{0}}^{\prime}$ has orbit disjoint from $p_{1}, \ldots, p_{k}$. Define $p_{j}^{\prime}=p_{j}$ for $j \neq j_{0}$ to obtain the new heteroclinic cycle $p_{1}^{\prime}, \ldots, p_{k}^{\prime}$. By $(2.2), d\left(\beta_{G}\left(m, p_{j_{0}}^{\prime}\right), \beta_{G}(m, x)\right) \leq C C_{5} \delta^{\alpha}$ (independent of the period $m$ of $p_{j_{0}}^{\prime}$ ). Choosing $\delta$ sufficiently small, we obtain

$$
d\left(\beta_{G}\left(m, p_{j_{0}}^{\prime}\right), \hat{g}\right)<r /(3 K) .
$$

Let $H_{j}^{\prime}$ denote the holonomies for the cycle $p_{1}^{\prime}, \ldots, p_{k}^{\prime}$ and set $\bar{H}_{j}^{\prime}=H_{1}^{\prime} \ldots H_{j-1}^{\prime}$. For $\delta$ small enough, we ensure that $d\left(\bar{H}_{j}^{\prime}, \bar{H}_{j}\right)$ is as small as required for all $j$ (in fact at most two of the $H_{j}$ are changed).

For $j \neq j_{0}$, we compute using the properties of the metric $d$ that

$$
\begin{aligned}
d\left(\bar{\beta}_{j}^{\prime}, \bar{\beta}_{j}\right) & =d\left(\bar{H}_{j}^{\prime} \beta_{j}\left(P_{j}\right) \bar{H}_{j}^{\prime-1}, \bar{H}_{j} \beta_{j}\left(P_{j}\right) \bar{H}_{j}^{-1}\right) \\
& \leq d\left(\bar{H}_{j}^{\prime-1}, \bar{H}_{j}^{-1}\right)+d\left(\bar{H}_{j}^{\prime} \beta_{j}\left(P_{j}\right) \bar{H}_{j}^{-1}, \bar{H}_{j} \beta_{j}\left(P_{j}\right) \bar{H}_{j}^{-1}\right) \\
& \leq d\left(\bar{H}_{j}^{\prime}, \bar{H}_{j}\right)\left\|\operatorname{Ad}\left(\bar{H}_{j}^{-1}\right)\right\|+d\left(\bar{H}_{j}^{\prime}, \bar{H}_{j}\right)\left\|\operatorname{Ad}\left(\beta\left(P_{j}\right) \bar{H}^{-1}\right)\right\| \leq \operatorname{Cd}\left(\bar{H}_{j}^{\prime}, \bar{H}_{j}\right),
\end{aligned}
$$

where $C$ is a constant that depends only on $\beta$ and the original heteroclinic cycle. Part (i) follows for $\delta$ sufficiently small. Letting $h^{\prime}$ denote the $G$-component of $\bar{H}_{j_{0}}^{\prime}$ we choose $\delta$ small so that $d\left(h^{\prime}, h\right), d\left(h^{\prime-1}, h^{-1}\right)<r /(3 K)$. Then

$$
\begin{aligned}
d\left(g_{j_{0}}^{\prime}, \bar{g}\right) & =d\left(h^{\prime} \beta_{G}\left(m, p_{j_{0}}^{\prime}\right) h^{\prime-1}, h \hat{g} h^{-1}\right) \\
& \leq d\left(h^{\prime} \beta_{G}\left(m, p_{j_{0}}^{\prime}\right) h^{\prime-1}, h^{\prime} \hat{g} h^{\prime-1}\right)+d\left(h^{\prime} \hat{g} h^{\prime-1}, h^{\prime} \hat{g} h^{-1}\right)+d\left(h^{\prime} \hat{g} h^{-1}, h \hat{g} h^{-1}\right) \\
& \leq K d\left(\beta_{G}\left(m, p_{j_{0}}^{\prime}\right), \hat{g}\right)+d\left(h^{\prime-1}, h^{-1}\right)+K d\left(h^{\prime}, h\right)<r,
\end{aligned}
$$

establishing part (ii).

\section{Class I Groups}

In this section, we prove Theorem 1.2 for groups of class I. 
6.1. Condition (2) of Theorem 4.1. The first step is to construct an open and dense set of cocycles with periodic heteroclinic cycles satisfying condition (2) of Theorem 4.1 .

Let $S^{n-1}$ denote the unit sphere in $\mathbb{R}^{n}$. If $P \subset \mathbb{R}^{n}$, we let co $P$ denote the convex hull of $P$.

Proposition 6.1. Suppose that $G$ acts on $\mathbb{R}^{n}$ with $\operatorname{Fix} G=\{0\}$, and that $y_{1}, \ldots, y_{n} \in$ $\mathbb{R}^{n}$ are linearly independent. Then for any $e \in S^{n-1}$ there exist $\bar{g}_{ \pm} \in G$ and $y \in$ $\left\{y_{1}, \ldots, y_{n}\right\}$ such that $\left\langle\bar{g}_{+} y, e\right\rangle>0$ and $\left\langle\bar{g}_{-} y, e\right\rangle<0$.

Proof. Choose $y$ such that $\langle y, e\rangle \neq 0$. We suppose for definiteness that $\langle y, e\rangle>0$ and take $\bar{g}_{+}=e_{G}$. If $\langle g y, e\rangle \geq 0$ for all $g \in G$, then $\langle\bar{y}, e\rangle>0$ where $\bar{y}=\int_{G} g y d \nu$ and $\nu$ is Haar measure. It follows from invariance of $\nu$ that $\bar{y} \in \operatorname{Fix} G=\{0\}$ which is a contradiction. Hence there exists $\bar{g}_{-} \in G$ such that $\left\langle\bar{g}_{-} y, e\right\rangle<0$.

Lemma 6.2. Suppose that $G$ acts on $\mathbb{R}^{n}$ with Fix $G=\{0\}$. Let $y_{i j} \in \mathbb{R}^{n}, 1 \leq i \leq n$, $1 \leq j \leq n+1$, and suppose that for each $j$, the vectors $y_{1 j}, \ldots, y_{n j}$ are linearly independent. Then there exist $\bar{g}_{i j} \in G, 1 \leq i \leq n, 1 \leq j \leq n+1$, such that $0 \in \operatorname{co}\left\{\bar{g}_{i j} y_{i j}\right\}$.

Proof. Let $M=n(n+1)$. For each $M$-tuple $\left\{\bar{g}_{i j}\right\} \in G^{M}$, we associate the convex set $K_{\bar{g}}=\operatorname{co}\left\{\bar{g}_{i j} y_{i j}\right\}$. The map from $G^{M}$ to convex sets $K_{\bar{g}}$ is continuous. Let $d=$ $\min _{\bar{g} \in G^{M}} \operatorname{dist}\left\{K_{\bar{g}}, 0\right\}$ and suppose for contradiction that $d>0$. Choose $\bar{g} \in G^{M}$ and $x \in K_{\bar{g}}$ such that $d(x, 0)=d$. Since $x \in \partial K_{\bar{g}}$, there exist $h_{1}, \ldots, h_{n} \in\left\{\bar{g}_{i j}\right\}$ with corresponding $z_{1}, \ldots, z_{n} \in\left\{y_{i j}\right\}$ such that $x \in \operatorname{co}\left\{h_{\ell} z_{\ell}\right\}$. There is at least one linearly independent $n$-tuple $\left\{y_{1 j^{*}}, \ldots, y_{n j^{*}}\right\}$ that does not include any of the $z_{\ell}$. By Proposition 6.1, we can redefine one of the $\bar{g}_{i j^{*}}$ so that $\left\langle\bar{g}_{i j^{*}} y_{i j^{*}}, x\right\rangle<0$. The new convex set $K^{\prime}$ contains $x$ and intersects the hyperplane orthogonal to $x$. It follows that $\operatorname{dist}\left(K^{\prime}, 0\right)<d$ yielding the required contradiction.

Recall that when dealing with Euclidean groups, we are given pairs $(g, v)$, with $v=w \oplus w^{\prime}$ where $w \in$ Fix $g$.

Proposition 6.3. Suppose that $\mathbb{R}^{n}$ is a $G$-irreducible representation of class $I$. Then for any $g_{1}, \ldots, g_{n} \in G$ and any $\delta>0$ there exist $h_{i} \in B_{\delta}\left(e_{G}\right)$ and $z_{i} \in h_{i}$ Fix $g_{i}$ such that $\left\{z_{1}, \ldots, z_{n}\right\}$ is a basis for $\mathbb{R}^{n}$.

Proof. Inductively, for $k<n$, suppose we have chosen $h_{i} \in B_{\delta}\left(e_{G}\right)$ and $z_{i} \in h_{i}$ Fix $g_{i}$, $i=1, \ldots, k$, such that $\left\{z_{1}, \ldots, z_{k}\right\}$ are linearly independent. Let $Z=\mathbb{R}\left\{z_{1}, \ldots, z_{k}\right\}$. Since $G$ acts irreducibly and Fix $g_{k+1} \neq\{0\}$, the set $G$ Fix $g_{k+1}$ spans $\mathbb{R}^{n}$ and hence there exists $\hat{h} \in G$ such that $\hat{h}$ Fix $g_{k+1} \not \subset Z$. Write $\hat{h}=\exp \eta$ where $\eta \in L G$, and let $h(t)=\exp t \eta$. If $h(t) \operatorname{Fix} g_{k+1} \subset Z$ for all $t$ close to zero, then differentiating repeatedly at $t=0$, we obtain $\eta^{n}$ Fix $g_{k+1} \subset Z$ for all integers $n \geq 0$. In particular $\hat{h}$ Fix $g_{k+1} \subset Z$ which contradicts the choice of $\hat{h}$. It follows that we can choose $h_{k+1}=$ $h\left(t_{0}\right) \in B_{\delta}\left(e_{G}\right)$ so that $h_{k+1}$ Fix $g_{k+1} \not \subset Z$. Now choose $z_{k+1} \in\left(h_{k+1}\right.$ Fix $\left.g_{k+1}\right) \backslash Z$.

Corollary 6.4. Let $G \ltimes \mathbb{R}^{n}$ be a Euclidean-type group of class I. Then for any $\left(g_{1}, v_{1}\right), \ldots,\left(g_{n+1}, v_{n+1}\right) \in G \ltimes \mathbb{R}^{n}$ and $\delta>0$, there exist $\left(\tilde{g}_{i}, \tilde{v}_{i}\right) \in B_{\delta}\left(g_{i}, v_{i}\right)$ such that $\operatorname{co}\left\{\tilde{w}_{1}, \ldots, \tilde{w}_{n+1}\right\}$ has nonempty interior. 
Proof. Writing $\mathbb{R}^{n}=V_{1} \oplus \cdots \oplus V_{s}$, we let $d_{\ell}=\operatorname{dim} V_{\ell}, \ell=1, \ldots, s$, so that $d_{1}+\cdots+d_{s}=$ $n$. By Proposition 6.3, there exist $h_{i} \in B_{\delta / 2}\left(e_{G}\right)$ and $z_{i} \in h_{i}$ Fix $g_{i} \cap V_{1}, i=1, \ldots, d_{1}$, such that $\left\{z_{1}, \ldots, z_{d_{1}}\right\}$ is a basis for $V_{1}$. Similarly, there exist $h_{i} \in B_{\delta / 2}\left(e_{G}\right)$ and $z_{i} \in h_{i}$ Fix $g_{i} \cap V_{2}, i=d_{1}+1, \ldots, d_{1}+d_{2}$, such that $\left\{z_{d_{1}+1}, \ldots, z_{d_{1}+d_{2}}\right\}$ is a basis for $V_{2}$. Continuing in this way, we obtain $h_{i} \in B_{\delta / 2}\left(e_{G}\right)$ and $z_{i} \in h_{i}$ Fix $g_{i}, i=1, \ldots, n$, such that $\left\{z_{1}, \ldots, z_{n}\right\}$ is a basis for $\mathbb{R}^{n}$.

Let $\tilde{g}_{i}=h_{i} g_{i} h_{i}^{-1}$ and $\tilde{v}_{i}=h_{i} v_{i}$ with corresponding vectors $\tilde{w}_{i}=h_{i} w_{i} \in$ Fix $\tilde{g}_{i}=$ $h_{i}$ Fix $g_{i}$. The next step is to perturb so that $\left\{\tilde{w}_{1}, \ldots, \tilde{w}_{n}\right\}$ is a basis for $\mathbb{R}^{n}$. Define $\tilde{w}_{i}(\varepsilon)=\tilde{w}_{i}+\varepsilon z_{i}$. Note that $P(\varepsilon)=\operatorname{det}\left(\tilde{w}_{1}(\varepsilon)|\ldots| \tilde{w}_{n}(\varepsilon)\right)$ is a polynomial of order $n$ in $\varepsilon$ and the coefficient of $\varepsilon^{n}$ is $\operatorname{det}\left(z_{1}|\ldots| z_{n}\right)$ which is nonzero. Hence $P\left(\varepsilon_{0}\right) \neq 0$ for some $\varepsilon_{0} \in(0, \delta / 2)$ and we obtain elements $\left(\tilde{g}_{i}, \tilde{v}_{i}+\varepsilon_{0} z_{i}\right) \in B_{\delta}\left(g_{i}, v_{i}\right)$ with corresponding vectors $\tilde{w}_{i}+\varepsilon_{0} z_{i} \in \operatorname{Fix}\left(\tilde{g}_{i}\right)$ forming a basis for $\mathbb{R}^{n}$. Relabelling, we may suppose that $\left\{\tilde{w}_{1}, \ldots, \tilde{w}_{n}\right\}$ is a basis for $\mathbb{R}^{n}$.

Finally, if necessary (i) perturb $v_{n+1}$ so that $w_{n+1} \neq 0$ and (ii) rescale $v_{n+1}$ to $\tilde{v}_{n+1}=$ $(1+\lambda) v_{n+1}$ with $|\lambda|<\delta$ so that the rescaled $\tilde{w}_{n+1}$ does not belong to $\operatorname{co}\left\{\tilde{w}_{1}, \ldots, \tilde{w}_{n}\right\}$.

Lemma 6.5. Let $G \ltimes \mathbb{R}^{n}$ be a Euclidean-type group of class $I$. Set $L=n(n+1)^{2}$. For any $\left(g_{1}, v_{1}\right), \ldots,\left(g_{L}, v_{L}\right) \in G \ltimes \mathbb{R}^{n}$ and any $\delta>0$, there exist $\left(\tilde{g}_{i}, \tilde{v}_{i}\right) \in B_{\delta}\left(g_{i}, v_{i}\right)$ and $\bar{g}_{i} \in G, i=1, \ldots, L$, such that $0 \in \operatorname{Int} \operatorname{co}\left\{\bar{g}_{1} \tilde{w}_{1}, \ldots, \bar{g}_{L} \tilde{w}_{L}\right\}$.

Proof. Relabel the pairs $\left(g_{i}, v_{i}\right)$ as $\left(g_{i j k}, v_{i j k}\right)$ where $1 \leq i \leq n, 1 \leq j, k \leq n+1$. For each $i, j$ consider the $(n+1)$-tuple $k=1, \ldots, n+1$. Let $K_{i j}=\operatorname{co}\left\{w_{i j k}, 1 \leq k \leq n+1\right\}$. Applying Corollary 6.4. we may suppose after a $\delta$-small perturbation that each $K_{i j}$ has nonempty interior.

For each $j$, choose $y_{i j} \in \operatorname{Int} K_{i j}, i=1, \ldots, n$, such that $\left\{y_{1 j}, \ldots, y_{n j}\right\}$ is a basis for $\mathbb{R}^{n}$. By Lemma 6.2, there exist $\bar{g}_{i j} \in G, 1 \leq i \leq n, 1 \leq j \leq n+1$, such that $0 \in \operatorname{co}\left\{\bar{g}_{i j} y_{i j}\right\}_{i, j}$. Hence $0 \in \operatorname{Int} \operatorname{co}\left\{\bar{g}_{i j} w_{i j k}\right\}_{i, j, k}$.

Write $\beta: X \rightarrow \Gamma$ as $\beta=\left(\beta_{G}, \beta_{\mathbb{R}^{n}}\right): X \rightarrow G \ltimes \mathbb{R}^{n}$. Recall from Section 4 that for periodic heteroclinic cycles $p_{1}, \ldots, p_{k}$ in $X$ and for $k$-tuples $\left(n_{1}, \ldots, n_{k}\right)$, there is an associated set of vectors $\left\{u_{1}, \ldots, u_{k+1}\right\} \subset \mathbb{R}^{n}$. Condition (2) of Theorem 4.1 requires that $0 \in Z\left(u_{1}, \ldots, u_{k+1}\right)=\left\{\alpha_{1} u_{1}+\cdots+\alpha_{k+1} u_{k+1}: \alpha_{1}, \ldots, \alpha_{k+1}>0\right\}$. It suffices that $0 \in \operatorname{Int} \operatorname{co}\left\{u_{2}, \ldots, u_{k}\right\}$ which is moreover a stable condition.

Theorem 6.6. Let $G \ltimes \mathbb{R}^{n}$ be a Euclidean-type group of class I. Assume that the compact group extension $f_{\beta_{G}}: X \times G \rightarrow X \times G$ is stably transitive.

Let $p_{1}$ be a periodic point for $f: X \rightarrow X$. Suppose that $p_{1}, \ldots, p_{k}$ is a periodic heteroclinic cycle in $X$ with $k \geq 2 L+2$, where $L=n(n+1)^{2}$.

Then there exist

(i) a cocycle $\tilde{\beta}$ that is arbitrarily close to $\beta$ in the $C^{r}$ topology and such that $\tilde{\beta}-\beta$ is supported in an arbitrarily small neighborhood of the points $p_{2}, \ldots, p_{k}$; and

(ii) a periodic heteroclinic cycle $p_{1}^{\prime}=p_{1}, p_{2}^{\prime}, \ldots, p_{k}^{\prime}$ with $p_{j}^{\prime}$ arbitrarily close to $p_{j}$ for $j=1, \ldots, k$;

such that the set $\left\{u_{1}, \ldots, u_{k+1}\right\}$ corresponding to $p_{1}^{\prime}, p_{2}^{\prime}, \ldots, p_{k}^{\prime}$ has the property that $0 \in \operatorname{Int} \operatorname{co}\left\{u_{2}, \ldots, u_{k}\right\}$. 
Proof. Let $\bar{\beta}_{j}=\left(g_{j}, v_{j}\right)$ be the data for the cycle $p_{1}, \ldots, p_{k}$ with associated vectors $w_{j} \in \operatorname{Fix} g_{j}$. Set $j_{i}=2 i+1, i=1, \ldots, L$. By Lemma 6.5, there exist arbitrarily small perturbations of $\left(g_{j}, v_{j}\right)$ and there exist $\bar{g}_{1}, \ldots, \bar{g}_{L} \in G$ such that $0 \in \operatorname{Int} \operatorname{co}\left\{\bar{g}_{1} w_{j_{1}}, \ldots, \bar{g}_{L} w_{j_{L}}\right\}$.

Since $X \times G$ is transitive, by Lemma 5.1 we can choose a new heteroclinic cycle $p_{1}^{\prime}=p_{1}, p_{2}^{\prime}, \ldots, p_{k}^{\prime}$, allowing us to specify $g_{j_{i}-1}^{\prime}$ whilst keeping $\bar{\beta}_{j_{i}}^{\prime}=\left(g_{j_{i}}^{\prime}, v_{j_{i}}^{\prime}\right)$ almost unchanged. (The cocycle $\beta$ is unchanged.) Recall that $u_{j_{i}}^{\prime}=h_{j_{i}}^{\prime} w_{j_{i}}^{\prime}$ where $h_{j_{i}}^{\prime}=$ $g_{1}^{\prime} \ldots g_{j_{i}-1}^{\prime}$. By specifying $g_{j_{i}-1}^{\prime}$ appropriately, we can arrange that $h_{j_{i}}^{\prime}$ is as close to $\bar{g}_{i}$ as desired. Since $w_{j_{i}}^{\prime}$ is almost unchanged, we can ensure that $0 \in \operatorname{Int} \operatorname{co}\left\{u_{j_{1}}^{\prime}, \ldots, u_{j_{m}}^{\prime}\right\}$.

6.2. Proof of Theorem $\mathbf{1 . 2}$ for class I groups. By Theorem 2.5 and Remark $1.3(\mathrm{~b})$, it suffices to perturb $\beta$ so that $\mathcal{L}_{\beta}\left(p_{1}\right)$ contains elements that generate $\Gamma$ as a closed semigroup.

By [2], the compact cocycle $\beta_{G}: X \rightarrow G$ is transitive for an open dense set of $C^{r}$ cocycles $\beta: X \rightarrow \Gamma$. Hence, we may suppose without loss that $\beta_{G}$ is stably transitive.

Pick a periodic point $p_{1}$. By Theorem 6.6, there is a periodic heteroclinic cycle starting at $p_{1}$ and an arbitrarily small perturbation of $\beta$ such that $0 \in \operatorname{Int} \operatorname{co}\left\{u_{2}, \ldots, u_{k}\right\}$. This condition is stable under perturbation. Since the perturbation is localised, and using stability of transitivity of $\beta_{G}$, we can construct a second periodic heteroclinic cycle starting at $p_{1}$ with $0 \in \operatorname{Int} \operatorname{co}\left\{u_{2}^{\prime}, \ldots, u_{k}^{\prime}\right\}$.

We have established condition (2) of Theorem 4.1 in a stable manner. A further small perturbation of $\beta_{G}$ guarantees that condition (1) is also satisfied. Hence, by Theorem 4.1, we obtain two elements $A_{1}, A_{2} \in \mathcal{L}_{\beta}\left(p_{1}\right)$.

Write $A_{i}=\left(g_{i}, v_{i}\right)$. Since $G$ is compact, there exists $g_{1}^{\prime}, g_{2}^{\prime}$ arbitrarily close to $g_{1}, g_{2}$ such that the closed group, and hence the closed semigroup, generated by $g_{1}^{\prime}, g_{2}^{\prime}$ is the whole of $G$ [11. We show that there is an arbitrarily small perturbation of $\beta$ that perturbs $g_{1}, g_{2}$ to $g_{1}^{\prime}, g_{2}^{\prime}$.

The elements $A_{1}, A_{2}$ are computed according to 4.3$)$ :

$$
A=\beta\left(P_{1}\right)^{n_{1}} H_{1} \beta\left(P_{2}\right)^{2 n_{2}} H_{2} \cdots \beta\left(P_{k}\right)^{2 n_{k}} H_{k} \beta\left(P_{1}\right)^{n_{k+1}} .
$$

We consider perturbations localized along the heteroclinic connections. The holonomies $H_{j}$ are given by explicit formulas in Lemmas 2.6 and 3.2 , and depend continuously on $\beta$. To change in a prescribed manner, say, only $H_{k}$, while keeping all the other $H_{j} \mathrm{~s}$ and the $\beta\left(P_{i}\right)$ s fixed, it suffices to change $\beta$ near only one point on the heteroclinic orbit from $p_{k}$ to $p_{k+1}=p_{1}$. The elements $\bar{\beta}_{j}$ are modified slightly as a result but only via conjugation, so condition (1) in Theorem 4.1 is maintained, while our construction for condition (2) is stable and hence maintained. The effect of this change on the $A_{i} \mathrm{~s}$ is described by (4.3). Hence, we obtain slightly modified elements $A_{1}^{\prime}, A_{2}^{\prime} \in \mathcal{L}_{\beta}\left(p_{1}\right)$, and we can arrange by this process that $g_{1}, g_{2}$ be perturbed to $g_{1}^{\prime}, g_{2}^{\prime}$, as desired.

Now we perturb again in order to generate the $\mathbb{R}^{n}$-part of $\Gamma$. By Corollary 4.2, it suffices to arrange that the closed semigroup generated by $\mathcal{U}=\left\{u_{2}, \ldots, u_{k}\right\}$ is the whole of $\mathbb{R}^{n}$. Moreover, $\mathcal{U}$ does not lie in a halfspace (since $0 \in \operatorname{Int} c o \mathcal{U}$ ) so it follows from Niţică \& Pollicott [8] or [6, Lemma 2.12], that it suffices to arrange that the closed 
group generated by $\mathcal{U}=\left\{u_{2}, \ldots, u_{k}\right\}$ is the whole of $\mathbb{R}^{n}$. Thus, (using Kronecker's theorem) it suffices to have $\left\{\tilde{u}_{1}, \ldots, \tilde{u}_{n+1}\right\} \subset \mathcal{U}$ such that these $(n+1)$ vectors generate $\mathbb{R}^{n}$ over $\mathbb{R}$, and writing $\sum_{1}^{n+1} \alpha_{j} \tilde{u}_{j}=0$, the scalars $\left\{\alpha_{j} \mid 1 \leq j \leq n+1\right\}$ are independent over $\mathbb{Q}$.

Recall that the elements of $\mathcal{U}$ are described by formula 4.2$)$. Because $0 \in \operatorname{Int} \operatorname{co} \mathcal{U}$, it contains at least $(n+1)$ nonzero vectors that span $\mathbb{R}^{n}$ over $\mathbb{R}$. To make the $\mathbb{Z}$-span of $\mathcal{U}$ dense in $\mathbb{R}^{n}$, we rescale the $\mathbb{R}^{n}$-component of the cocycle $\beta$ near the periodic orbits $P_{j}$, while keeping $\beta_{G}$ unchanged. This yields $w_{j} \mapsto\left(1+\delta_{j}\right) w_{j}$. From formula 4.2, one sees that the effect on $\mathcal{U}$ is $u_{j} \mapsto\left(1+\delta_{j}\right) u_{j}$.

Since $\mathcal{L}_{\beta}\left(p_{1}\right)$ is closed, it follows from Corollary 4.2 that $\mathbb{R}^{n} \cdot A_{1} \subset \mathcal{L}_{\beta}\left(p_{1}\right)$ and $\mathbb{R}^{n} \cdot A_{2} \subset \mathcal{L}_{\beta}\left(p_{1}\right)$. Thus $\left(g_{i}, 0\right) \in \mathcal{L}_{\beta}\left(p_{1}\right), i=1,2$, and consequently $G \times\{0\} \subset \mathcal{L}_{\beta}\left(p_{1}\right)$. Therefore $\left\{e_{G}\right\} \times \mathbb{R}^{n} \subset \mathcal{L}_{\beta}\left(p_{1}\right)$, so $\Gamma \subset \mathcal{L}_{\beta}\left(p_{1}\right)$.

\section{Proof of Theorem 1.2}

In this section, we complete the proof of Theorem 1.2. In Subsection 7.1, we consider the case where there are summands of class II in addition to the summands of class I. In Subsection 7.2, we consider the general case.

7.1. Summands of class II. Next suppose that $\mathbb{R}^{n}$ is a mixture of representations of class I and II. Then $\mathbb{R}^{n}=W_{1} \oplus$ Fix $G$ where $W_{1}$ is a sum of irreducible representations of class I. Let $\pi_{1}: \mathbb{R}^{n} \rightarrow W_{1}$ and $\pi: \mathbb{R}^{n} \rightarrow$ Fix $G$ be the associated projections. Let $d_{1}=\operatorname{dim} W_{1}, d=\operatorname{dim} \operatorname{Fix} G, d_{1}+d=n$.

We generalise Theorem 6.6 as follows:

Theorem 7.1. Let $G \ltimes \mathbb{R}^{n}$ be a Euclidean-type group with summands of class $I$ and II. Assume that the compact group extension $f_{\beta_{G}}: X \times G \rightarrow X \times G$ is stably transitive. Assume further that $\pi \beta: X \rightarrow$ Fix $G$ is not cohomologous to a cocycle with values in a halfspace.

Set $L=d_{1}(d+1)(n+1)^{2}, k=2 L+2$, and let $p_{1}$ be a periodic point for $f: X \rightarrow X$. Then there exist

(i) a periodic heteroclinic cycle $p_{1}, \ldots, p_{k}$ in $X$;

(ii) a cocycle $\tilde{\beta}$ that is arbitrarily close to $\beta$ in the $C^{r}$ topology and such that $\tilde{\beta}-\beta$ is supported in an arbitrarily small neighborhood of the points $p_{2}, \ldots, p_{k}$; and

(iii) a periodic heteroclinic cycle $p_{1}^{\prime}=p_{1}, p_{2}^{\prime}, \ldots, p_{k}^{\prime}$ with $p_{j}^{\prime}$ arbitrarily close to $p_{j}$ for $j=1, \ldots, k$;

such that the set $\left\{u_{1}, \ldots, u_{k+1}\right\}$ corresponding to $p_{1}^{\prime}, p_{2}^{\prime}, \ldots, p_{k}^{\prime}$ has the property that $0 \in \operatorname{Int} \operatorname{co}\left\{u_{2}, \ldots, u_{k}\right\}$.

Moreover, there are arbitrarily many heteroclinic cycles of this type that are disjoint, except for the common point $p_{1}$.

In the remainder of this subsection, we prove Theorem 7.1. As usual, to each $(g, v) \in G \ltimes \mathbb{R}^{n}$, there is an associated vector $w \in$ Fix $g$. Note that $\pi v=\pi w$.

Proposition 7.2. Let $y_{i j} \in \mathbb{R}^{n}, 1 \leq i \leq d_{1}, 1 \leq j \leq d+1$. Suppose that

(a) For each $i$ the set $\left\{\pi y_{i j}: 1 \leq j \leq d+1\right\}$ does not lie in a halfspace in Fix $G$; 
(b) Any collection of $d_{1}$ vectors in $\left\{\pi_{1} y_{i j}\right\}_{i, j}$ is a linearly independent subset of $W_{1}$.

Then for any $e \in S^{n-1}$, there exist $y_{ \pm} \in\left\{y_{i j}\right\}_{i, j}$ and $\bar{g}_{ \pm} \in G$ such that $\left\langle\bar{g}_{+} y_{+}, e\right\rangle>0$ and $\left\langle\bar{g}_{-} y_{-}, e\right\rangle<0$.

Proof. Assume first that $\pi e \neq 0$. By assumption (a), for each $i$ there exists $y_{i \pm} \in$ $\left\{y_{i j}: 1 \leq j \leq d+1\right\}$ such that $\left\langle\pi y_{i+}, \pi e\right\rangle>0$ and $\left\langle\pi y_{i-}, \pi e\right\rangle<0$. By assumption (b), the vectors $\left\{\pi_{1} y_{1+}, \ldots \pi_{1} y_{d_{1}+}\right\}$ are linearly independent in $W_{1}$. By Proposition 6.1. there exists $y_{+} \in\left\{y_{1+}, \ldots, y_{d_{1}+}\right\}$ and $\bar{g}_{+} \in G$ such that $\left\langle\pi_{1} \bar{g}_{+} y_{+}, \pi_{1} e\right\rangle>0$. It follows that $\left\langle\bar{g}_{+} y_{+}, e\right\rangle>0$.

Similarly $\left\{\pi_{1} y_{1-}, \ldots \pi_{1} y_{d_{1}-}\right\}$ are linearly independent in $W_{1}$, and so there exist $y_{-}$ and $\bar{g}_{-}$with the desired properties.

If $\pi e=0$ the above conclusion still holds, ignoring the Fix $G$ components.

Lemma 7.3. Let $y_{i j k} \in \mathbb{R}^{n}, 1 \leq i \leq d_{1}, 1 \leq j \leq d+1,1 \leq k \leq n+1$. Suppose that

(a) For each $i, k$ the set $\left\{\pi y_{i j k}: 1 \leq j \leq d+1\right\}$ does not lie in a halfspace in Fix $G$;

(b) For each $k$, any collection of $d_{1}$ vectors in $\left\{\pi_{1} y_{i j k}\right\}_{i, j}$ is a linearly independent subset of $W_{1}$.

Then there exist $\bar{g}_{i j k} \in G$ such that $0 \in \operatorname{co}\left\{\bar{g}_{i j k} y_{i j k}\right\}_{i, j, k}$.

Proof. Let $M=d_{1}(d+1)(n+1)$. For each $M$-tuple $\left\{\bar{g}_{i j k}\right\} \in G^{M}$, we associate the convex set $K_{\bar{g}}=\operatorname{co}\left\{\bar{g}_{i j k} y_{i j k}\right\}$. The map from $G^{M}$ to convex sets $K_{\bar{g}}$ is continuous. Let $d=\min _{\bar{g} \in G^{M}} \operatorname{dist}\left\{K_{\bar{g}}, 0\right\}$ and suppose for contradiction that $d>0$. Choose $\bar{g} \in G^{M}$ and $x \in K_{\bar{g}}$ such that $d(x, 0)=d$. Since $x \in \partial K_{\bar{g}}$, there exist $h_{1}, \ldots, h_{n} \in\left\{\bar{g}_{i j k}\right\}_{i, j, k}$ with corresponding $\tilde{y}_{1}, \ldots, \tilde{y}_{n} \in\left\{y_{i j k}\right\}_{i, j, k}$ such that $x \in \operatorname{co}\left\{h_{\ell} \tilde{y}_{\ell}\right\}$. There is at least one $d_{1}(d+1)$-tuple $\left\{y_{i j k^{*}}: 1 \leq i \leq d_{1}, 1 \leq j \leq d+1\right\}$ that does not include any of the $\tilde{y}_{\ell}$. Assumptions (a) and (b) in the statement of the lemma translate into the corresponding hypotheses for Proposition 7.2, and hence we can redefine one of the $\bar{g}_{i j k^{*}}$ so that $\left\langle\bar{g}_{i j k^{*}} y_{i j k^{*}}, x\right\rangle<0$. The new convex set $K^{\prime}$ contains $x$ and intersects the hyperplane orthogonal to $x$. It follows that $\operatorname{dist}\left(K^{\prime}, 0\right)<d$ yielding the required contradiction.

Proposition 7.4. Let $\left(g_{1}, v_{1}\right), \ldots,\left(g_{n+1}, v_{n+1}\right) \in G \ltimes \mathbb{R}^{n}$ and $\delta>0$. Suppose that $\left\{\pi v_{1}, \ldots, \pi v_{n+1}\right\}$ does not lie in a closed halfspace in Fix $G$. Then there exist $\left(\tilde{g}_{i}, \tilde{v}_{i}\right) \in$ $B_{\delta}\left(g_{i}, v_{i}\right)$ such that $\operatorname{co}\left\{\tilde{w}_{1}, \ldots, \tilde{w}_{n+1}\right\}$ has nonempty interior.

Proof. Without loss, we may suppose that $\left\{\pi w_{d_{1}+1}, \ldots, \pi w_{n}\right\}$ is a basis for Fix $G$. As in Proposition 6.3, we can perturb $g_{1}, \ldots, g_{d_{1}}$ slightly (by conjugation) so that there is a basis $\left\{z_{1}, \ldots, z_{d_{1}}\right\}$ for $W_{1}$ formed of vectors $z_{i} \in \operatorname{Fix} g_{i} \cap W_{1}$. Define $\tilde{w}_{i}(\varepsilon)=w_{i}+\varepsilon z_{i}$ for $1 \leq i \leq d_{1}$ and $\tilde{w}_{i}(\varepsilon)=w_{i}$ for $d_{1}+1 \leq i \leq n$. The coefficient of $\varepsilon^{d_{1}}$ in $\operatorname{det}\left(\tilde{w}_{1}(\varepsilon)|\ldots| \tilde{w}_{n}(\varepsilon)\right)$ is given by $\operatorname{det}\left(z_{1}|\ldots| z_{d_{1}}\right) \times \operatorname{det}\left(\pi w_{d_{1}+1}|\ldots| \pi w_{n}\right) \neq 0$. Hence we can choose $\tilde{w}_{i}=\tilde{w}_{i}\left(\varepsilon_{0}\right)$ with $\varepsilon_{0}$ arbitrarily small such that $\left\{\tilde{w}_{1}, \ldots, \tilde{w}_{n}\right\}$ is a basis for $\mathbb{R}^{n}$. Finally, perturb/scale $w_{n+1}$ slightly if necessary to obtain the required result. 
Proposition 7.5. Suppose that $\pi \beta: X \rightarrow$ Fix $G$ is not cohomologous to a cocycle with values in a halfspace. Then

(i) There exist periodic orbits $P_{1}, \ldots, P_{d+1} \in X$ such that the vectors $\pi \beta\left(P_{1}\right), \ldots$, $\pi \beta\left(P_{d+1}\right)$ do not lie in a halfspace in Fix $G$.

(ii) Set $v_{\ell}^{*}=\pi \beta\left(P_{\ell}\right) /\left|\pi \beta\left(P_{\ell}\right)\right|, \ell=1, \ldots, d+1$. Then for any $k \geq 1$ and any $\varepsilon>0$, there exist periodic orbits $P_{i \ell}$ with disjoint orbits, $1 \leq i \leq k, 1 \leq \ell \leq d+1$, such that $\pi \beta\left(P_{i \ell}\right) /\left|\pi \beta\left(P_{i \ell}\right)\right| \in B_{\varepsilon}\left(v_{\ell}^{*}\right)$ for all $i, \ell$.

Proof. The positive Livšic theorem of Bousch [1, Section 4] and the compactness of the set of hyperplanes imply that there are finitely many periodic orbits $P_{j}^{\prime}, 1 \leq j \leq F$, such that the set $\left\{\pi \beta\left(P_{j}^{\prime}\right): 1 \leq j \leq F\right\}$ does not lie in a halfspace of Fix $G$, and thus $0 \in \operatorname{Int} \operatorname{co}\left\{\pi \beta\left(P_{j}^{\prime}\right): 1 \leq j \leq F\right\}$. For any non-negative integers $n_{j}$ that are not all zero, one can use shadowing arguments (see, for example [8, Sections 5 and 6]) to obtain periodic orbits $Q_{n}$ such that $\pi \beta\left(Q_{n}\right)=n\left(\sum_{j=1}^{F} n_{j} \pi \beta\left(P_{j}^{\prime}\right)\right)+O(1)$.

Thus, one can obtain $d+1$ periodic orbits $P_{\ell}$ such that $0 \in \operatorname{Int} \operatorname{co}\left\{\pi \beta\left(P_{\ell}\right): 1 \leq \ell \leq\right.$ $d+1\}$, proving (i). Given one of the periodic orbits $P_{\ell}$ in (i), the above shadowing arguments yield a sequence of periodic orbits $Q_{n}$ with $\pi \beta\left(Q_{n}\right)=n \pi \beta\left(P_{\ell}\right)+O(1)$, proving (ii).

Proof of Theorem 7.1. We focus attention first on the $L$ periodic points $p_{3}, p_{5}, \ldots, p_{2 L+1}$, relabelling these as $p_{i j k \ell}, 1 \leq i \leq d_{1}, 1 \leq j \leq d+1,1 \leq k, \ell \leq n+1$. Let $\bar{\beta}_{i j k \ell}=\left(g_{i j k \ell}, v_{i j k \ell}\right)$ be the data for the cycle. By Proposition 7.5, we can choose the periodic points $p_{i j k \ell}$, unit vectors $v_{1}^{*}, \ldots, v_{d+1}^{*} \in \operatorname{Fix} G$, and $\varepsilon>0$, such that

- $v_{1}^{\prime}, \ldots, v_{d+1}^{\prime}$ do not lie in a halfspace for all $v_{j}^{\prime} \in B_{\varepsilon}\left(v_{j}^{*}\right) \cap \operatorname{Fix} G, 1 \leq j \leq d+1$; and

- $\pi v_{i j k \ell} /\left|\pi v_{i j k \ell}\right| \in B_{\varepsilon / 2}\left(v_{j}^{*}\right)$ for all $i, j, k, \ell$.

Let $K_{i j k}=\operatorname{co}\left\{w_{i j k \ell}: 1 \leq \ell \leq n+1\right\}$. By Proposition 7.4, we can perturb so that co $K_{i j k}$ has nonempty interior. Choose $y_{i j k} \in \operatorname{Int} K_{i j k}$ satisfying the requirement that whenever $1 \leq j \leq d+1$ we have $\pi y_{i j k} /\left|\pi y_{i j k}\right| \in B_{\varepsilon / 2}\left(\pi v_{i j k j} /\left|\pi v_{i j k j}\right|\right) \subset B_{\varepsilon}\left(v_{j}^{*}\right)$. Then condition (a) of Lemma 7.3 is satisfied. Modify the choices if necessary so that condition (b) is also satisfied. By Lemma 7.3, there exist $\bar{g}_{i j k \ell} \in G$ such that $0 \in \operatorname{Int} \operatorname{co}\left\{\bar{g}_{i j k \ell} w_{i j k \ell}\right\}$.

Next, we consider the $L$ periodic points $p_{2}, p_{4}, \ldots, p_{2 L}$, relabelling them as $q_{i j k \ell}$ (with corresponding periodic orbit $Q_{i j k \ell}$ ), where $q_{i j k \ell}$ is the periodic point immediately preceding $p_{i j k \ell}$. Since $X \times G$ is transitive, by Lemma 5.1 we can choose a new heteroclinic cycle $p_{1}^{\prime}=p_{1}, p_{2}^{\prime}, \ldots, p_{k}^{\prime}$, allowing us to specify $\beta_{G}\left(Q_{i j k \ell}\right)$ whilst keeping the data $\left(g_{i j k \ell}, v_{i j k \ell}\right)$ for $p_{i j k \ell}$ almost unchanged. (The cocycle $\beta$ is unchanged.) As in the proof of Theorem 6.6. we can ensure that $0 \in \operatorname{Int} \operatorname{co}\left\{u_{3}^{\prime}, \ldots, u_{2 L+1}^{\prime}\right\}$.

7.2. The general case. Finally, we consider the general case with summands of classes I, II and III. Write $\mathbb{R}^{n}=W_{1} \oplus W_{2} \oplus$ Fix $G$ and let $d_{1}=\operatorname{dim} W_{1}, d_{2}=\operatorname{dim} W_{2}$, $d=\operatorname{dim} \operatorname{Fix} G, d_{1}+d_{2}+d=n$.

Denote $G_{0}=\left\{g \in G \mid \operatorname{Fix}(g) \cap W_{2}=\{0\}\right\}$. By the following Proposition 7.6, the set $G_{0}$ is open and dense in $G$. 
Proposition 7.6. Suppose that $\mathbb{R}^{n}$ is a $G$-irreducible representation of class III. Then the set $U=\{g \in G:$ Fix $g=\{0\}\}$ is open and dense in $G$.

Proof. Note that $g \in U$ if and only if 1 is not an eigenvalue which is an open condition. By the definition of class III, there exists $g_{0} \in U$. Let $\mathbb{T}$ denote a maximal torus in $G$ containing $g_{0}$. Then Fix $\mathbb{T}=\{0\}$. Moreover Fix $g=\{0\}$ for any generator $g$ of $\mathbb{T}$. The set of elements of $G$ that generate maximal tori is dense and hence $U$ is dense.

We generalise Theorems 6.6 and 7.1 as follows:

Theorem 7.7. Let $G \ltimes \mathbb{R}^{n}$ be a general Euclidean-type group. Assume that the compact group extension $f_{\beta_{G}}: X \times G \rightarrow X \times G$ is stably transitive. Assume further that $\pi \beta: X \rightarrow$ Fix $G$ is not cohomologous to a cocycle with values in a halfspace.

Set $L=d_{1}(d+1)\left(d_{1}+d+1\right)^{2}, k=2 L+2$ and let $p_{1}$ be a periodic point for $f: X \rightarrow X$. Then there exist

(i) a periodic heteroclinic cycle $p_{1}, \ldots, p_{k}$ in $X$;

(ii) a cocycle $\tilde{\beta}$ that is arbitrarily close to $\beta$ in the $C^{r}$ topology and such that $\tilde{\beta}-\beta$ is supported in an arbitrarily small neighborhood of the points $p_{2}, \ldots, p_{k}$; and

(iii) a periodic heteroclinic cycle $p_{1}^{\prime}=p_{1}, p_{2}^{\prime}, \ldots, p_{k}^{\prime}$ with $p_{j}^{\prime}$ arbitrarily close to $p_{j}$ for $j=1, \ldots, k$;

such that for any cocycle sufficiently $C^{r}$-close to $\tilde{\beta}$, the set $\left\{u_{1}, \ldots, u_{k+1}\right\}$ corresponding to $p_{1}^{\prime}, p_{2}^{\prime}, \ldots, p_{k}^{\prime}$ has the property that $0 \in Z\left(u_{2}, \ldots, u_{k}\right)$.

Moreover, there are arbitrarily many heteroclinic cycles of this type that are disjoint, except for the common point $p_{1}$.

Proof. Let $\hat{\pi}: \mathbb{R}^{n} \rightarrow W_{1} \oplus$ Fix $G$ be the orthogonal projection onto summands of class I and II, and let $\pi_{2}: \mathbb{R}^{n} \rightarrow W_{2}$ be the complementary projection. Let $\bar{\beta}_{j}=\left(g_{j}, v_{j}\right)$ be the data for the cycle $p_{1}, \ldots, p_{k}$ with associated vectors $w_{j} \in$ Fix $g_{j}$.

By Theorem 7.1, we can make the choices in (i), (ii) and (iii), so that $0 \in$ Int $\operatorname{co}\left\{\hat{\pi} u_{2}, \ldots, \hat{\pi} u_{k}\right\} \subset W_{1} \oplus$ Fix $G$ where $\left\{u_{1}, \ldots, u_{k+1}\right\}$ is the set corresponding to $p_{1}^{\prime}, \ldots, p_{k}^{\prime}$. In particular, $0 \in Z\left(\hat{\pi} u_{2}, \ldots, \hat{\pi} u_{k}\right)$ and this condition is stable to further perturbations of the cocycle $\tilde{\beta}$.

By Proposition 7.6, we can perturb $\tilde{\beta}$ if necessary so that each $g_{j}$ lies in the open dense subset $G_{0}$. For each $j$, we have $\pi_{2} w_{j}=0$ and so $\pi_{2} u_{j}=0$. It follows that $Z\left(\pi_{2} u_{2}, \ldots, \pi_{2} u_{k}\right)=\{0\}$ in a stable manner. Hence $0 \in Z\left(u_{2}, \ldots, u_{k}\right)$ and this condition is stable to further perturbations of the cocycle $\tilde{\beta}$.

Let $\Gamma_{2}=G \ltimes W_{2}$ be the Euclidean-type group corresponding to the summands of class III.

Proposition 7.8. The set of $\left(d_{2}+3\right)$-tuples in $\Gamma_{2}^{d_{2}+3}$ that generate $\Gamma_{2}$ as a closed semigroup is residual.

Proof. This follows from [4, Equation (2.1) and Proposition 2.4].

Proof of Theorem 1.2. We repeat the approach of Subsection 6.2, starting with Theorem 7.7 instead of Theorem 6.6. By Theorem 4.1 we can construct $d_{2}+3$ elements 
$A_{j}=\left(g_{j}, v_{j}\right)$ in $\mathcal{L}_{\beta}\left(p_{1}\right)$ that can be perturbed independently and vary continuously with the cocycle $\beta$. As in Subsection 6 , we rescale locally the $W_{1} \oplus$ Fix $G$-components of the cocycle to obtain from Corollary 4.2 that $\left(W_{1} \oplus\right.$ Fix $\left.G\right) \cdot A_{j} \subset \mathcal{L}_{\beta}\left(p_{1}\right)$ for each

$A_{j}$. Thus we have elements $A_{j}^{\prime}=\left(g_{j}, v_{j}^{\prime}\right) \in \mathcal{L}_{\beta}\left(p_{1}\right)$ with $v_{j}^{\prime} \in W_{2}$. Use Proposition 7.8 to perturb the $G \ltimes W_{2}$-component of the cocycle so that $G \ltimes W_{2} \subset \mathcal{L}_{\beta}\left(p_{1}\right)$. Thus $W_{1} \oplus$ Fix $G \subset \mathcal{L}_{\beta}\left(p_{1}\right)$, and therefore $\mathcal{L}_{\beta}\left(p_{1}\right)=\Gamma$.

\section{REFERENCES}

[1] T. Bousch. La condition de Walters, Ann. Sci. Ecole. Norm. Sup. 34 (2001) 287-311.

[2] M. Field, I. Melbourne, A. Török. Stable ergodicity for smooth compact Lie group extensions of hyperbolic basic sets. Ergod. Th. \& Dynam. Sys. 25 (2005) 517-551.

[3] A. Katok, B. Hasselblatt. Introduction to the modern theory of dynamical systems, Cambridge University Press, 1995.

[4] I. Melbourne, M. Nicol. Stable transitivity of Euclidean group extensions. Ergod. Th. \& Dynam. Sys. 23 (2003) 611-619.

[5] I. Melbourne, V. Niţică, A. Török. Stable transitivity of certain noncompact extensions of hyperbolic systems. Annales Henri Poincaré 6 (2005) 725-746.

[6] I. Melbourne, V. Niţică, A. Török. A note about stable transitivity of noncompact extensions of hyperbolic systems . Discrete Contin. Dynam. Systems 14 (2006) 355-363.

[7] S. Newhouse. Lectures on dynamical systems. Dynamical Systems, Progress in Mathematics, 8, Birkhäuser, Basel, 1-114, 1980.

[8] V. Niţică, M. Pollicott. Transitivity of Euclidean extensions of Anosov diffeomorphisms. Ergod. Th. \& Dynam. Sys. 25 (2005) 257-269.

[9] V. Niţică, A. Török. An open and dense set of stably ergodic diffeomorphisms in a neighborhood of a non-ergodic one. Topology 40 (2001) 259-278.

[10] M. Pollicott, C. P. Walkden. Livšic theorems for connected Lie groups. Trans. Amer. Math. Soc. 353 (2001) 2879-2895.

[11] J. Schreier, S. Ulam. Sur le nombre de generateurs d'un groupe topologique compact et connexe. Fund. Math. 24 (1935) 302-304.

IM: Department of Mathematics and Statistics, University of Surrey, Guildford, SURREY GU2 7XX, UK

E-mail address: ism@math.uh.edu

VN: Department of Mathematics, 323 Anderson Hall, West Chester University, West Chester, PA 19383, and Institute of Mathematics of the Romanian Academy, P.O. Box 1-764, RO-70700 Bucharest, Romania

E-mail address: vnitica@wcupa.edu

AT: University of Houston, Department of Mathematics, 651 PGH, Houston, TX 77204-3008, and Institute of Mathematics of the Romanian Academy, P.O. Box 1-764, RO-70700 Bucharest, Romania

E-mail address: torok@math.uh.edu 